\title{
Interaction of Argon Clusters with Intense VUV-Laser Radiation: The Role of Electronic Structure in the Energy-Deposition Process
}

\author{
T. Laarmann, ${ }^{1, *}$ A. R. B. de Castro, ${ }^{2}$ P. Gürtler, ${ }^{1}$ W. Laasch,,${ }^{1}$ J. Schulz, ${ }^{1, \dagger}$ H. Wabnitz, ${ }^{1}$ and T. Möller ${ }^{1}$ \\ ${ }^{1}$ Hamburger Synchrotronstrahlungslabor HASYLAB at Deutsches Elektronen Synchrotron DESY, \\ Notkestrasse 85, 22607 Hamburg, Germany \\ ${ }^{2}$ Laboratorio Nacional de Luz Sincrotron, 13084-971 Campinas SP, Brazil and Instituto de Fisica Gleb Wataghin, \\ Universidade Estadual de Campinas, 13083-970 Campinas SP, Brazil
}

(Received 5 September 2003; published 9 April 2004)

\begin{abstract}
The response of Ar clusters to intense vacuum-ultraviolet pulses is investigated with photoion spectroscopy. By varying the laser wavelength, the initial excitation was either tuned to absorption bands of surface or bulk atoms of clusters. Multiple ionization is observed, which leads to Coulomb explosion. The efficiency of resonant 2-photon ionization for initial bulk and surface excitation is compared with that of the nonresonant process at different laser intensities. The specific electronic structure of clusters plays almost no role in the explosion dynamics at a peak intensity larger than $1.8 \times 10^{12} \mathrm{~W} / \mathrm{cm}^{2}$. The inner ionization of atoms for resonant and nonresonant excitation is then saturated and the energy deposition is mainly controlled by the plasma heating rate. Molecular dynamics simulations indicate that standard collisional heating cannot fully account for the strong energy absorption.
\end{abstract}

DOI: 10.1103/PhysRevLett.92.143401

Presently, there is a world wide effort to develop lasers for soft and hard $\mathrm{x}$ rays. Free-electron lasers (FEL) making use of linear accelerators are expected to provide short-wavelength radiation with laserlike properties, many orders of magnitude brighter than synchrotrons. Short-wavelength radiation emitted from FEL is delivered in pulses of a few tens to several hundred femtosecond (fs) length. Their unique properties promise to reveal structures of large biomolecules, visualize ultrafast chemical processes with atomic resolution, and produce plasmas in a very controlled way. The interaction of very intense FEL light with matter differs considerably from that of conventional sources. At optical frequencies much effort has gone into the understanding of the interaction of high-intensity fs-laser pulses with matter in the past decade. Clusters as a state of matter with intermediate size between molecules and solid targets have played an important role in this context. A variety of fascinating phenomena were observed, for instance, emission of coherent $\mathrm{x}$ rays [1], hot electrons, and energetic, highly charged ions [2,3]. Even investigation of deuterium fusion in clusters became feasible [4]. All these effects are due to the absorption of a large amount of energy by the cluster, resulting in the efficient ionization and subsequent explosion of the clusters.

In the first development phase (2000-2002), the FEL of the TESLA Test Facility (TTF) at DESY provided gigawatt pulses of 30-100 fs length below $100 \mathrm{~nm}$ wavelength [5]. This has opened the door to a new regime of strong-field matter interaction with photons capable to excite or ionize matter directly [6]. On the basis of simple scaling laws, it can be expected that strong-field matter interaction is considerably different in the vacuum-ultraviolet (VUV) and soft $\mathrm{x}$-ray regime from that at optical frequencies [7]. The ponderomotive energy $U_{p} \sim I / \omega^{2}$
PACS numbers: $36.40 . \mathrm{Gk}, 52.50 . \mathrm{Jm}$

(I, $\omega$ : intensity and frequency of the laser source), which is the average kinetic energy of a free electron oscillating in the radiation field, characterizes to some extent the interaction. For comparison, with intense VUV radiation from the TTF-FEL, one obtains an $U_{p}$ of less than $100 \mathrm{meV}$, whereas at typical optical fs-laser parameters $\left(800 \mathrm{~nm} ; 10^{16} \mathrm{~W} / \mathrm{cm}^{2}\right), U_{p}$ reaches values of several hundred $\mathrm{eV}$. In this context it is an interesting question whether the specific electronic structure of materials such as band structure has a strong effect on the light-matter interaction at short wavelength. More specifically, it is interesting to know if resonant, strongly absorbing states enhance the interaction.

In this Letter, we present experimental results on VUVlaser interaction with Ar clusters. The radiation wavelength is tunable in a wide range of 80-120 nm [5]. This wavelength regime covers the excitonic bulk and surface absorption bands of Ar clusters at $\sim 100$ and $\sim 105 \mathrm{~nm}$, respectively [8]. By varying the photon energy, resonant and nonresonant processes can be induced and the effect of the excitation energy on the energy absorption of $\mathrm{Ar}$ clusters can be investigated. The Ar clusters were prepared in a supersonic expansion through a $100 \mu \mathrm{m}$ conical nozzle with a half opening angle of $15^{\circ}$. The average cluster size $\langle N\rangle$ was tuned in the range between 170 and 900 atoms per cluster by varying the stagnation pressure according to well-known scaling laws $[9,10]$. The full width at half maximum $\Delta N$ of the size distribution is approximately $\langle N\rangle$. FEL radiation with a typical pulse energy between 0.3 and $5 \mu \mathrm{J}$ and a pulse duration of about $50 \mathrm{fs}$ was focused on the cluster beam using an elliptical mirror at grazing incidence. The pulse duration was estimated indirectly with statistical and spectral methods [5]. By measuring the homogeneous focal spot on a fluorescence screen with a microscope and a charge coupled 
divice camera, a diameter of $\sim 20 \mu \mathrm{m}$ was derived. As a result, a maximum peak intensity up to $\sim 10^{13} \mathrm{~W} / \mathrm{cm}^{2}$ was estimated. The ions produced during the interaction were detected with a microchannel plate (MCP) of a time-of-flight (TOF) mass spectrometer coupled to a digitizing oscilloscope. The distance between the interaction region and the MCP was $25 \mathrm{~mm}$. The TOF was operated using an extraction voltage of $3.4 \mathrm{kV}$. To investigate the dependence of the energy deposition on the specific electronic structure such as bulk and surface excitonic states [8], the laser wavelength was tuned to different values as indicated in the absorption spectrum of $\mathrm{Ar}_{800}$ clusters shown in Fig. 1. This spectrum was taken from a previous work using fluorescence excitation spectroscopy with synchrotron light [8]. By choosing 105, 100.8, and $96.6 \mathrm{~nm}$ as FEL wavelengths, site-selective excitation was assured although the spectral width is $0.5-1 \%$. This is important in order to separate the effect of different excitation schemes on the energy deposition process.

TOF mass spectra in Fig. 2(a) recorded at three different excitation energies and a peak intensity of $\sim 1.5 \times$ $10^{13} \mathrm{~W} / \mathrm{cm}^{2}$, show that $\mathrm{Ar}_{900}$ clusters completely disintegrate into multiply charged atomic fragments. Many photons are taken from the laser field. We would like to note that at least two FEL photons are needed to ionize an $\mathrm{Ar}$ atom because the ionization potential is $15.76 \mathrm{eV}$. From the flight times, the kinetic energy distribution of $\mathrm{Ar}^{q+}$ ionic fragments can be derived, which gives direct insight into the efficiency of the energy deposition. Surprisingly, at high peak intensity of $\sim 10^{13} \mathrm{~W} / \mathrm{cm}^{2}$, the ionization of clusters is independent of the wavelength and consequently not affected by the specific electronic structure of the primary excited state. The energy deposition due to surface $(105 \mathrm{~nm})$, bulk $(100.8 \mathrm{~nm})$, and nonresonant excitation $(96.6 \mathrm{~nm})$ is of similar efficiency, as seen by the identical kinetic energy distributions of $\mathrm{Ar}^{q+}$ [Fig. 2(a)]. In contrast, at a lower peak intensity of $\sim 10^{11} \mathrm{~W} / \mathrm{cm}^{2}$, which was obtained by moving the cluster beam $3 \mathrm{~mm}$

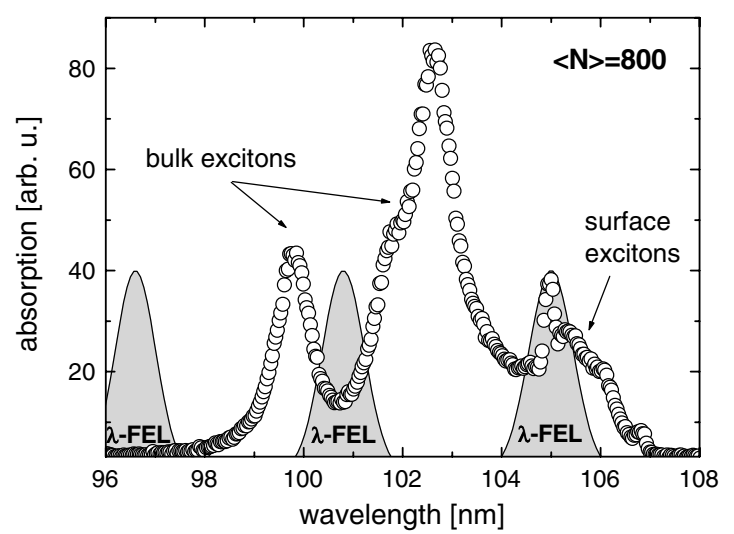

FIG. 1. Photoabsorption spectrum of $\mathrm{Ar}_{800}$ clusters [8]. As indicated, the FEL radiation wavelength was tuned to surface and bulk excitons as well as to nonresonant excitation. out of the focal spot, the mass spectra differ considerably [Fig. 2(b)]. Heavy cluster fragments $\left(\mathrm{Ar}^{2+}, \mathrm{Ar}^{3+}\right)$ increase in intensity for nonresonant excitation. We like to note that the "spiky" temporal structure of the FEL pulses does not seem to play a key role in the VUV-laser cluster interaction. The single-shot mass spectra for a given FEL wavelength and intensity look very similar, although the spikes are significantly changing on a shotto-shot basis [5]. This is presumably due to the large number of electronic degrees of freedom in clusters.

To help the understanding of the experimental results in more detail, we recall that Coulomb explosion of clusters is controlled by ionization and heating rate. During the energy deposition, a highly excited medium is formed. Meanwhile, the cluster undergoes different phases [11], namely, (i) inner ionization of the atoms forming a plasma, (ii) heating of the quasifree electrons, and (iii) outer ionization of the cluster by electron emission from the highly charged cluster resulting in (iv) the complete disintegration of the cluster. Therefore, the total energy absorbed during a laser pulse depends sensitively on the inner ionization rate because it controls the plasma density and consequently the plasma heating rate. In this picture the energy absorption of Ar clusters exposed to VUV-FEL pulses at a peak intensity of $\sim 1.5 \times$ $10^{13} \mathrm{~W} / \mathrm{cm}^{2}$ is mainly controlled by the plasma heating rate, whereas at a lower peak intensity of $\sim 1.9 \times$ $10^{11} \mathrm{~W} / \mathrm{cm}^{2}$ the limiting factor is the inner ionization rate at the beginning of the laser pulse. Here, the electronic structure of the clusters comes into play with large differences in the cross section for on- and off-resonant excitation. The population of $\mathrm{Ar}^{2+}$ and $\mathrm{Ar}^{3+}$ ions in the mass spectra is a signature of the totally absorbed energy. Above a critical number of absorbed photons, the integrated peak area of $\mathrm{Ar}^{2+}$ and $\mathrm{Ar}^{3+}$ drops because the cluster starts to fragment preferentially into atomic ions. In Fig. 3, their relative abundance in the mass spectra of

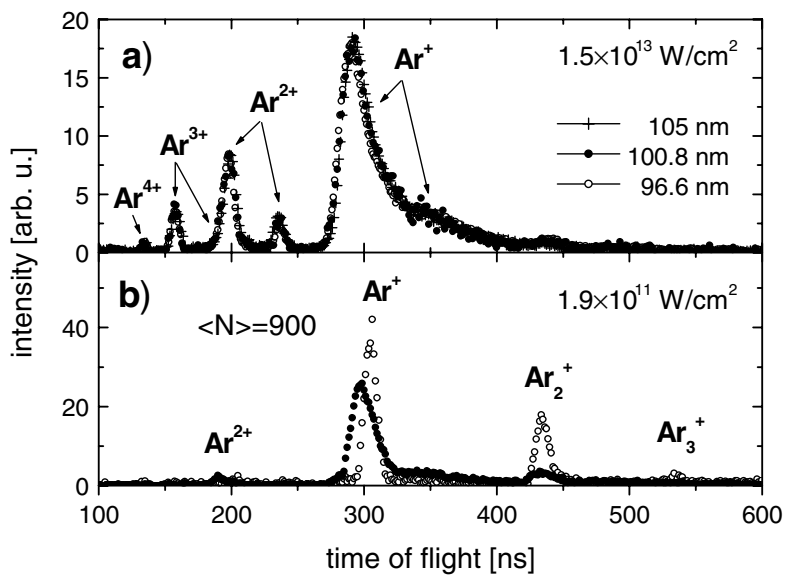

FIG. 2. TOF mass spectra recorded after irradiation of $\mathrm{Ar}_{900}$ clusters at three different wavelengths (surface, bulk, and nonresonant excitation) and peak intensity [(a) $1.5 \times 10^{13} \mathrm{~W} / \mathrm{cm}^{2}$ and (b) $1.9 \times 10^{11} \mathrm{~W} / \mathrm{cm}^{2}, 3 \mathrm{~mm}$ outside the focus]. 
$\mathrm{Ar}_{900}$ clusters is plotted as a function of the peak intensity for both initial bulk excitation and nonresonant excitation. Slightly above the saturation intensity of exciton absorption $I_{\mathrm{exc}} \sim 4 \times 10^{11} \mathrm{~W} / \mathrm{cm}^{2}$, resonant excitation leads to a complete disintegration of the clusters. The value of $I_{\mathrm{exc}}$ was estimated using the approximation of an "ideal" two-level system [12], taking into account the exciton absorption cross section of the bulk solid ( $\sim 100$ Mbarn) [13]. On the other hand, for nonresonant excitation with FEL pulses of the same intensity $I_{\mathrm{exc}}$, a significant contribution from dimer ions is still present in the mass spectrum. At somewhat higher peak intensity $\sim 1.8 \times 10^{12} \mathrm{~W} / \mathrm{cm}^{2}$, the two curves fall together, which means that strongly absorbing excitonic states do not enhance the inner ionization rate at larger peak intensity. Therefore, we assume that in this regime during the first few femtoseconds each atom in the Ar cluster becomes singly ionized by direct, simultaneous two-photon absorption. The transition probability for such a process depends mainly on the matrix element between the ground state of the cluster and the final ionized quasifree state. This gives a straight forward explanation for the observed effects in the limit of high intensities. After the initial ionization, a plasma is formed, which in the beginning consists of one quasifree electron per ion. The energy deposition is then mainly controlled by the absorption of a plasma that depends sensitively on the ion density and the average charge per ion. The quasifree electrons inside the cluster, which gain enough energy to overcome the potential barrier due to collisional heating, leave the dense cluster ion core by some kind of thermionic electron emission [6].

In order to determine the average charge state and the total energy absorbed by the cluster, we have simulated TOF mass spectra using an ion optics ray-tracing program. In the calculation of the ion trajectories, the measured center kinetic energy and width of each distribution $\left(\mathrm{Ar}^{+}-\mathrm{Ar}^{6+}\right)$ are taken as input parameters, and an iso-

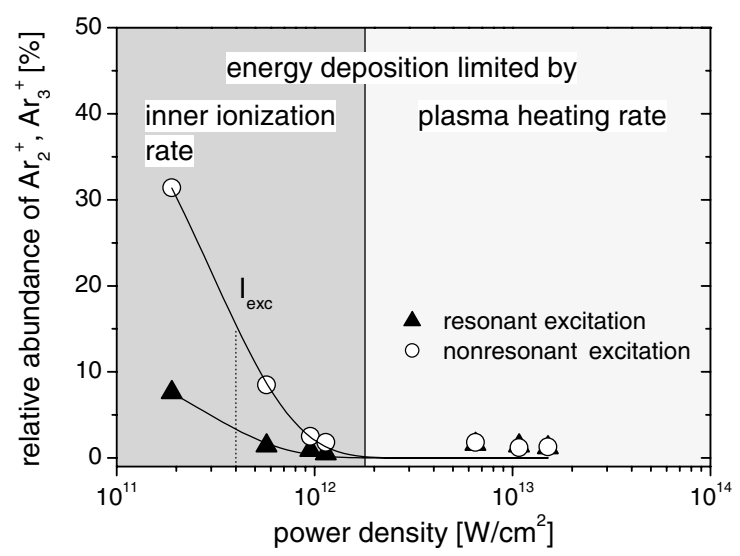

FIG. 3. Relative abundance of $\mathrm{Ar}_{2}^{+}, \mathrm{Ar}_{3}^{+}$resulting from irradiation of $\mathrm{Ar}_{900}$ clusters depending on the laser peak intensity. Initial resonant bulk excitation is compared with nonresonant excitation. tropic explosion of a fixed number of ions for each charge state is assumed. We obtained relative geometric acceptances $G_{q}$ of the spectrometer for different charge states $\mathrm{Ar}^{q+}$ and respective ejection energies $E_{q, e}$, but no information on the "real" charge distribution of the exploding cluster. Additionally, we took into account that the MCP-detection efficiency $\epsilon_{q}$ depends linearly on the velocity of the charged fragments [14]. The result of the simulation for Ar clusters comprising 170 atoms exposed to FEL radiation at an intensity of $3.2 \times 10^{13} \mathrm{~W} / \mathrm{cm}^{2}$ and initial bulk excitation at $100.8 \mathrm{~nm}$ is shown in Fig. 4(a). For comparison, the experimental result is presented in Fig. 4(b). The overall agreement between simulation and experiment is rather good, proving that the obtained $G_{q}$ values can be used for further analysis of the experimental data. Small differences are most probably due to non Gaussian-like kinetic energy distributions in the Coulomb explosion. We would like to note that the detected intensities of "backward peaks" are reduced compared to "forward peaks," which is presumably due to scattering effects in the interaction zone. Thus, we used slightly different intensity scalings in the simulation shown in Fig. 4(a). The average ejection energy $\bar{E}_{e}$ and charge state $\bar{Q}$ per ion are derived by weighing each integrated forward peak area $A_{q}$ in Fig. 4(b), corresponding to a specific charge $q$ and ejection energies $E_{q, e}$ with its respective relative geometric acceptance $G_{q}$ and the MCP-detection efficiency $\epsilon_{q}$. In the case of $\mathrm{Ar}_{170}$ clusters and the laser parameters given above, we obtain $\bar{E}_{e}=$ $240 \mathrm{eV}$ and $\bar{Q}=2$. This means on average each atom in the cluster loses two electrons and absorbs up to 20 photons during a $50 \mathrm{fs}$ long FEL pulse of high intensity. If we assume that the clusters are heated up by sequential single photon processes, this corresponds to an average cross section per atom of $\sigma_{\text {exp }}=24.1$ Mbarn.

To shed light on the basic mechanism of the absorption process in clusters in the VUV range, we performed

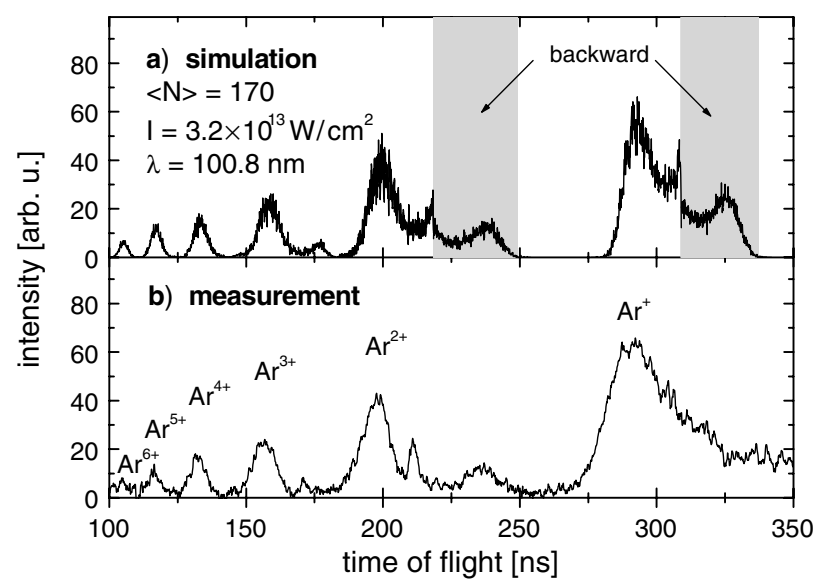

FIG. 4. Comparison between simulated (a) and measured (b) TOF mass spectra of irradiated $A_{170}$ clusters $(100.8 \mathrm{~nm}$, $\left.3.2 \times 10^{13} \mathrm{~W} / \mathrm{cm}^{2}\right) . \mathrm{Ar}^{+}$and $\mathrm{Ar}^{2+}$ ejected into the half-sphere facing away from the detector are indicated. 


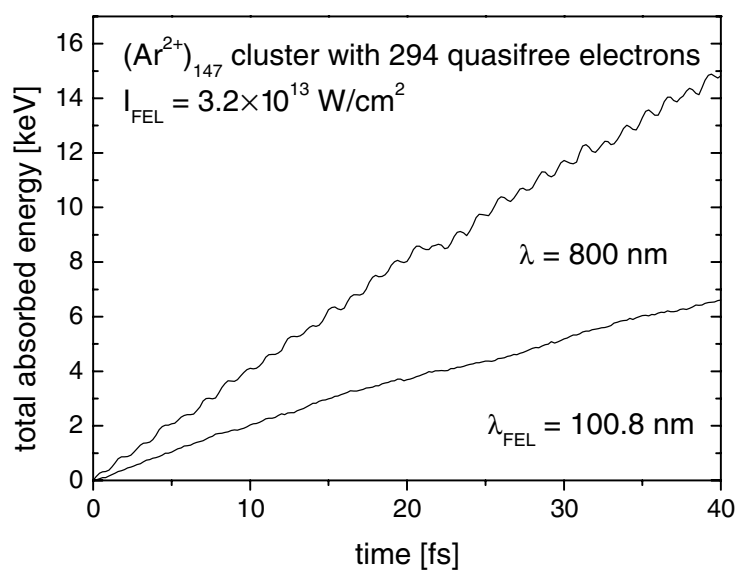

FIG. 5. Calculated energy absorption of the $\operatorname{Ar}_{147}$ cluster plasma containing two quasifree electrons per ion in the laser field $\left(3.2 \times 10^{13} \mathrm{~W} / \mathrm{cm}^{2}\right.$ for both 100.8 and $\left.800 \mathrm{~nm}\right)$.

molecular dynamics simulations of classical electron motion under the action of an intense laser pulse [15], according to the model of Last and Jortner [16]. The model naturally includes collisional heating effects of quasifree electrons and ions inside the cluster, such as inverse bremsstrahlung and plasma resonance absorption [17]. We start the simulation with two quasifree electrons per ion $(\bar{Q}=2)$ inside Ar clusters comprising 147 atoms (closed-shell icosahedron). The electrons are propagated as point charges under the influence of the Coulomb forces and the laser field. For the Coulomb potential, a neutral ground state structure of the cluster is assumed [16]. The temporal development of the total absorbed energy of 294 electrons moving under the influence of the laser field at $3.2 \times 10^{13} \mathrm{~W} / \mathrm{cm}^{2}$ and $100.8 \mathrm{~nm}$ is given in Fig. 5. For comparison with previous experiments at longer wavelength and as a cross check, the simulation for $800 \mathrm{~nm}$ is included in the figure. At long wavelength, i.e., low frequency, the electrons can follow the laser field more easily and can efficiently absorb energy due to collisional heating. At $100.8 \mathrm{~nm}$ the field is oscillating with a frequency that is much higher than the characteristic frequency of the electron gas [18]. Therefore, the absorption due to classical scattering processes is significantly reduced in the short-wavelength limit. At $100.8 \mathrm{~nm}$, the graph shows almost a linear increase with time. From the slope we estimated an absorption cross section of $\sigma_{\text {sim }}=$ 5.8 Mbarn, which is significantly smaller than the experimental value of $\sigma_{\text {exp }}=24.1 \mathrm{Mbarn}$. It is important to mention that the calculation overestimates the cross section, since it starts already with quasifree electrons inside the cluster and does not take the cluster expansion into account.

We would like to note that the discrepancy between experiment and simulation might be solved by recent theoretical work on Xe clusters by Santra and Greene [19]. They extended the "standard" model of inverse Bremsstrahlung mainly by including a more realistic atomic potential in the scattering process of electrons and ions compared to the commonly used Coulomb approach in collisional heating theory. Because of the reduced screening of the nuclear charge, they obtain significantly enhanced absorption in the VUV-laser cluster interaction in agreement with the experimental results.

In conclusion, $\operatorname{Ar}_{N}$ clusters $(N \leq 900)$ exposed to intense vacuum-ultraviolet FEL pulses of a few $10^{12} \mathrm{~W} / \mathrm{cm}^{2}$ completely disintegrate into multiply charged atomic ions by Coulomb explosion. In particular, strongly absorbing electronically excited states of clusters (surface and bulk excitons) do not enhance the total energy deposition compared to nonresonant excitation. This indicates that in this intensity regime the absorption is mainly controlled by plasma heating, and that at the beginning of the laser pulse two-photon inner ionization is saturated for resonant as well as for nonresonant twophoton processes. Each Ar atom absorbs up to 20 photons and loses on average 2 electrons. Molecular dynamics simulations show that standard collisional heating cannot fully account for the strong energy absorption. On the other hand, at lower peak intensity $\left(I<10^{12} \mathrm{~W} / \mathrm{cm}^{2}\right)$, the total energy deposition is controlled by the twophoton inner ionization rate, which differs considerably for initial resonant and nonresonant excitation.

We thank A. Swiderski for technical support and the TTF team at DESY for providing the FEL beam and the photon diagnostics. This work was supported by DFG.

*Present address: Max-Born-Institute, Max-Born Strasse 2a, D-12489 Berlin, Germany.

Electronic address: tim.laarmann@desy.de

${ }^{\dagger}$ Present address: MAX-lab, Box 118, 22100 Lund, Sweden.

[1] A. McPherson et al., Nature (London) 370, 631 (1994).

[2] T. Ditmire et al., Nature (London) 398, 489 (1999).

[3] E. Springate et al., Phys. Rev. A 61, 063201 (2000).

[4] T. Ditmire et al., Nature (London) 386, 54 (1997).

[5] V. Ayvazyan et al., Eur. Phys. J. D 20, 149 (2002).

[6] H. Wabnitz et al., Nature (London) 420, 482 (2002).

[7] U. Saalmann et al., Phys. Rev. Lett. 89, 143401 (2002).

[8] J. Wörmer et al., J. Chem. Phys. 104, 8269 (1996).

[9] O. F. Hagena, Z. Phys. D 4, 291 (1987).

[10] U. Buck et al., J. Chem. Phys. 105, 5408 (1996).

[11] V. P. Krainov et al., Phys. Rep. 370, 237 (2002).

[12] W. Demtröder, in Laserspektroskopie (Springer, Berlin, 1999), 4th ed.

[13] R. Haensel et al., J. Phys. (France) 32, C4 (1971).

[14] G.W. Fraser, Int. J. Mass Spectrom. 215, 13 (2002).

[15] J. Schulz et al., Nucl. Instrum. Methods Phys. Res. A 507, 572 (2003).

[16] I. Last and J. Jortner, Phys. Rev. A 62, 13201 (2000).

[17] A. Brantov et al., Phys. Plasmas 10, 3385 (2003).

[18] I. Last and J. Jortner, Phys. Rev. A 60, 2215 (1999).

[19] R. Santra and C. Greene, Phys. Rev. Lett. 91, 233401 (2003). 